\title{
Monitoring gas hydrate formation with magnetic resonance imaging in a metallic core holder
}

\author{
Mojtaba Shakerian 1,*, Armin Afrough ${ }^{1}$, Sarah Vashaee ${ }^{1}$, Florin Marica1 ${ }^{1}$, Yuechao Zhao ${ }^{2}$, Jiafei Zhao ${ }^{2}, Y_{0}$ gchen Song $^{2}$, \\ and Bruce J. Balcom ${ }^{1}$
}

${ }^{1}$ MRI Center, University of New Brunswick, Fredericton, NB, E3B 5A3, Canada

${ }^{2}$ Key Laboratory of Ocean Energy Utilization and Energy Conservation of the Ministry of Education, Dalian University of Technology, Dalian, 116024, China

\begin{abstract}
Methane hydrate deposits world-wide are promising sources of natural gas. Magnetic Resonance Imaging (MRI) has proven useful in previous studies of hydrate formation. In the present work, methane hydrate formation in a water saturated sand pack was investigated employing an MRI-compatible metallic core holder at low magnetic field with a suite of advanced MRI methods developed at the UNB MRI Centre. The new MRI methods are intended to permit observation and quantification of residual fluids in the pore space as hydrate forms. Hydrate formation occurred in the water-saturated sand at 1500 psi and $4{ }^{\circ} \mathrm{C}$. The core holder has a maximum working pressure of 4000 psi between -28 and $80{ }^{\circ} \mathrm{C}$. The heat-exchange jacket enclosing the core holder enabled very precise control of the sample temperature. A pure phase encode MRI technique, SPRITE, and a bulk T1-T2 MR method provided high quality measurements of pore fluid saturation. Rapid 1D SPRITE MRI measurements time resolved the disappearance of pore water and hence the growth of hydrate in the sand pack. $3 \mathrm{D} \pi$-EPI images confirmed that the residual water was inhomogeneously distributed along the sand pack. Bulk T1-T2 measurements discriminated residual water from the pore gas during the hydrate formation. A recently published local T1-T2 method helped discriminate bulk gas from the residual fluids in the sample. Hydrate formation commenced within two hours of gas supply. Hydrate formed throughout the sand pack, but maximum hydrate was observed at the interface between the gas pressure head and the sand pack. This irregular pattern of hydrate formation became more uniform over 24 hours. The rate of hydrate formation was greatest in the first two hours of reaction. An SE-SPI T2 map showed the T2 distribution changed considerably in space and time as hydrate formation continued. Changes in the T2 distribution are interpreted as pore level changes in residual water content and environment.
\end{abstract}

\section{Introduction}

Methane hydrates, in permafrost and oceanic deposits, are promising sources of energy. Hydrate formation is a complex process that can alter the pore structure and mechanical properties of the reservoir [1]. Considerable motivation exists for investigation of hydrate formation and dissociation in sediments to enhance gas production from such deposits.

Magnetic Resonance (MR) and Magnetic Resonance Imaging (MRI) are non-destructive techniques that have proven useful for studying gas hydrates [2,3]. MR/MRI techniques, in principle, can directly quantify the residual water saturation, discriminate the residual water from pore gas, and characterize pore level behavior in situ, but this has not been yet demonstrated. High field MRI instruments have been previously employed for hydrate studies, but low field instruments are advantageous since they minimize susceptibility effects [4]. At low static fields, appropriate MRI methods can spatially and temporally quantify residual fluids [5] (water and gas) and reveal the growth pattern of gas hydrate.

In recent hydrate publications, researchers employed glass bead samples to minimize magnetic susceptibility effects at 9.4 T [6,7]. Conventional frequency encode MRI measurements demonstrated that heat transfer to the sample region affects the hydrate dissociation process. Hydrate formation is exothermic. 3D images, obtained with conventional frequency encoded measurements, did not reveal the dynamic growth pattern of hydrate formation along the sand pack at $4.7 \mathrm{~T}$ [8].

Stevens [3] and Ersland [2] utilized 1D and 3D frequency encoding MRI methods to image methane hydrate formation. They measured a Bentheimer core plug at 2 Tesla static magnetic field with an echo time of $3 \mathrm{~ms}$ [2]. Each 3D image was acquired in one to nine hours [2]. The MRI methods employed did not discriminate possible residual gas from the residual water in the porous media during hydrate formation. 
Previous studies reported significant MRI signal loss as hydrate formed. Moderate $T_{2}$ signal lifetimes [6,7] did not permit monitoring the early and late stages of hydrate formation. In the present work, gas hydrate formation was monitored with advanced phase and frequency encoded MR/MRI measurements at $0.2 \mathrm{~T}$ in a metallic core holder $[9,10]$. Hydrate formation occurred in the water-saturated sand pack at $4{ }^{\circ} \mathrm{C}$ and 1500 psi [1].

A quantitative pure phase encode MRI technique, SPRITE, was employed to spatially and temporally monitor pore fluid saturation. Rapid 1D SPRITE MRI measurements [4] mapped the growth pattern of hydrate in the sand pack. $\pi$ Echo Planar Imaging ( $\pi$-EPI) [11] measured the 3D distribution of the residual water and the pore gas saturation along the sand pack. Bulk $T_{1}-T_{2}$ measurements [12] discriminated residual water from pore gas during hydrate formation. Bulk $T_{1}-T_{2}$ and $T_{2}$ measurements are interpreted in terms of pore level changes in water saturation, pore gas content and pore environment.

\section{Experimental}

An MR/MRI compatible metallic core holder [9] was utilized for ${ }^{1} \mathrm{H}$ MRI measurements of hydrate formation at $8.52 \mathrm{MHz}$. The core holder was employed in the bore of a Maran DRX-HF, vertical magnet, operating at $0.2 \mathrm{~T}$ (Oxford Instruments Ltd., Oxford, UK). A 1 kW 3445 RF amplifier (TOMCO, Sydney, Australia) and a threeaxis magnetic gradient coil, driven by Techron (Elkhart, IN) 7782 gradient amplifiers, were employed.

A sand pack (sand, mesh -50+70, Aldrich, MO, US), with length $38 \mathrm{~mm}$, diameter $33 \mathrm{~mm}$ and a porosity of $44 \%$ was employed as the porous media. A distilled water solution, with $500 \mathrm{ppm}$ sodium dodecyl sulfate (SDS), (Fisher, ON, CA) [13] saturated the sand pack. The sand was saturated with 14.7 grams of water. The sand pack was contained in a sample vessel $(2.5 \mathrm{~mm}$ wall thickness, length $51 \mathrm{~mm}$ ) that was fabricated from PEEK. The PEEK vessel was immersed in the confining fluid that pressurized the core holder interior [9]. Fluorinert FC-43, (3M, MN, US) was employed as the confining fluid. A circulating jacket enclosing the core holder exterior [9] regulated the temperature of the confining fluid to $4^{\circ} \mathrm{C}$. An ISCO pump (Teledyne ISCO, NE, US) held the confining pressure at 1650 psi. Methane gas (Praxair Canada Inc, NB, CA) was supplied at 1500 psi during hydrate formation. The sample vessel outlet was blocked as illustrated in Figure 1(d). Low pressure methane gas was initially injected into the apparatus, then purged, to remove air.

The SPRITE MRI method, as a pure phase encoding technique, is largely insensitive to internal magnetic field gradients and eddy currents, as well as $T_{1}$ and $T_{2}$ relaxation times [4]. 1D dhk SPRITE images, Figure 1, were acquired with $64 \mathrm{k}$-space points in 7 mins with 16 signal averages. The RF pulse length was $9^{\circ}$ with a duration of $1.5 \mu \mathrm{s}$ at $100 \% \mathrm{RF}$ power. The encoding time, $\mathrm{t}_{\mathrm{p}}$, was $150 \mu \mathrm{s}$ with a repetition time, TR, of $2 \mathrm{~ms}$. A delay time of $13 \mathrm{~s}$, equal to $5 \times$ bulk gas $\mathrm{T}_{1}, 2.6 \mathrm{~s}$, was employed between each of the two halves of the k-space trajectory. This resulted in complete recovery of the sample longitudinal magnetization before acquisition for bulk gas, bulk water and the water in the pore space. The delay time of $13 \mathrm{~s}$ was not sufficient for complete recovery of the longitudinal magnetization of the pore gas during the first 26 hours after introduction of the gas. The field of view (FOV) was $96 \mathrm{~mm}$ with a nominal resolution of $1.5 \mathrm{~mm}$.

$\pi$-EPI is a fast $3 \mathrm{D}$ frequency encoding MRI method [11] with reduced gradient duty cycle and increased sensitivity compared to conventional MRI methods, yielding high quality 3D images in short acquisition times [11]. Each 3D $\pi$-EPI image required 60 mins with 16 signal averages. Individual $\pi$-EPI images were not smoothed. The nominal resolution was $1.4 \times 1.4 \times 1.3$ $\mathrm{mm}^{3}$. A delay time of $14 \mathrm{~s}$ was employed between each of the 16 interleaved k-space trajectories. The echo time was $3 \mathrm{~ms}$. Bulk $\mathrm{T}_{1}-\mathrm{T}_{2}$ measurements [12] required 50 mins. SE-SPI $T_{2}$ mapping [4], local $T_{1}-T_{2}$ [12] and $2 \mathrm{D}$ Spiral SPRITE [4] measurements were also employed to monitor the hydrate formation process. Bulk CPMG and inversion recovery measurements monitored pore level changes in situ during hydrate formation.

A series of rapid MR/MRI measurements were undertaken sequentially during the initial stages of hydrate formation. These measurements were 1D dhk SPRITE, bulk Free Induction Decay, and bulk CPMG. After the first four hours, bulk $\mathrm{T}_{1}-\mathrm{T}_{2}$ measurement was added to the series. These four measurements continued for the next ten days, with periodic 3D $\pi$-EPI, SE-SPI $T_{2}$ mapping, local $\mathrm{T}_{1}-\mathrm{T}_{2}$, and 2D SPRITE measurements undertaken at specific times. Bulk inversion recovery measurements were undertaken periodically throughout the ten day period.

Bulk CPMG $T_{2}$ measurement and bulk inversion recovery $T_{1}$ measurement revealed that the water (with SDS) saturated sand pack, before gas introduction, had $\mathrm{T}_{1}$ and $\mathrm{T}_{2}$ relaxation times of $875 \mathrm{~ms}$ and $510 \mathrm{~ms}$ respectively. $\pi$-EPI and SE-SPI $\mathrm{T}_{2}$ mapping measurements confirmed that the initial water saturation was homogeneous before introduction of the gas.

\section{Results and discussion}

The 1D dhk SPRITE [4] experiment was employed to monitor the longitudinal hydrate formation process. 1D 
dhk SPRITE profiles, Figure 1, spatially resolved the bulk gas in the reservoir head from water saturating the sand pack. Measurement parameters were chosen to yield ${ }^{1} \mathrm{H}$ density weighted images for bulk gas, bulk water and water in the pore space, but not for gas in the pore space during the first 26 hours of reaction. Bulk $\mathrm{T}_{1^{-}}$ $\mathrm{T}_{2}$ measurements show that during the first 26 hours, the pore gas $T_{1}$ is on the order of $10 \mathrm{~s}$. The delay time, $13 \mathrm{~s}$, suppressed the pore gas magnetization during the first 26 hours. However, the last five dhk SPRITE profiles in Figure $1(\mathrm{c})$ yield a ${ }^{1} \mathrm{H}$ density weighting. The pore gas $\mathrm{T}_{1}$ was on the order of 10 seconds shortly after hydrate formation commenced, but decreased to approximately four seconds after 26 hours, as seen in Figure 3. We discuss below our hypothesis that the long $T_{1}$ species observed is pore gas.

The 1D dhk SPRITE [4] profile images were readily able to monitor the system and observed a 2 hour induction period after introduction of the gas head. No change was observed in the dhk SPRITE profiles during the first two hours. A realistic porous media, sand, with an SDS surfactant in the water phase was employed to minimize the induction period [14]. Intensive gradient switching, causing vibration of the gradient coil and sample, seemed to catalyze hydrate formation. Gradient vibration was observed to catalyze hydrate formation in a replica experiment.

Application of the pressure head resulted in displacement of some water from the sand pack into threads sealing the bottom of the sample vessel. This bulk like water was observed at $85 \mathrm{~mm}$ in Figure 1(a). The threads are located below the sample chamber above the O-ring, as seen in Figure 1(d). This water is visible throughout the experiment; methane did not convert this water to hydrate. It thus acted as a convenient internal reference throughout the ten day experiment. Hydrate formation in the sand pack is presumed to have sealed this region of the sample such that methane gas did not penetrate to the threads. In a replica experiment a similar water penetration was observed, but hydrate formation occurred and the signal was eventually lost.

Methane hydrate formation commenced at the interface of the sand pack and gas head as revealed by Figure 1(b). Loss of signal represents water in the pore space which is lost to the image as hydrate forms. The methane hydrate, as a quasi-solid [1], has MR lifetimes which are not amenable to observation and quantification even with the SPRITE method.

The majority of water in the sand pack was converted to hydrate within four hours of introducing gas. The induction period was two hours. Hydrate formation became more uniform in space with additional time, Figures 1(b) and (c). From 2 hours to 2.25 hours after introduction of the gas, the water saturation decreased from $100 \%$ to $74 \%$. The water saturation decreased to $32 \%$ at 2.75 hours after introduction of the gas. Water saturations were calculated based on the signal integrated from the sand pack area in the 1D dhk SPRITE profiles, and referenced to the initial water saturated sand pack.
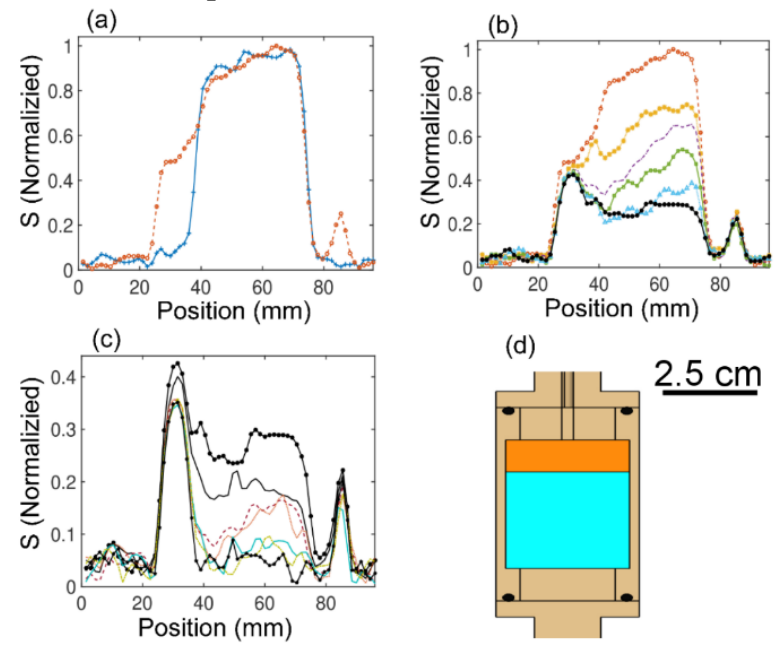

Fig. 1. Space and time resolved $1 \mathrm{H}$ fluid content profiles of hydrate formation. Methane penetrates the water saturated sand pack from a constant pressure gas head. Signal loss occurs when liquid water is converted to quasi solid hydrate. Hydrate forms first and most significantly near the gas head/sand pack interface. (a) profiles before and after introduction of the gas. The gas head is visible at left, $24 \mathrm{~mm}$ to $36 \mathrm{~mm}$, while a small amount of water is visible at right displaced from the sand pack into threads of the sample vessel below the sand pack. Observed signal intensity is normalized by the maximum signal in the time zero profile. (b) $1 \mathrm{H}$ fluid content profiles at 2 , $2.25,2.75,3.45,3.75$ and 4 hours after introduction of the gas. (c) $1 \mathrm{H}$ fluid content profile at 4, 14, 44, 68, 118, 152 and 217 hours after introduction of the gas. Hydrate formation has largely ceased approximately 120 hours after introduction of the gas but residual fluids, water and gas, remain in the pore space. Not the change in the y axis range in (c). (d) Schematic of the sample vessel. The gas head (orange region), connected to a constant pressure supply, is located on top of the water saturated sand layer (turquoise region).

It is well known that the rate of hydrate formation in idealized systems, pure liquid or ideal porous media such as glass beads, is much slower than in more realistic systems such as a sand pack [14]. This phenomenon was observed in the present experiment. Within four hours of introducing gas, $30 \%$ of bulk water in the methane reservoir was converted to hydrate whereas during the same period $70 \%$ of the water saturating the sand was converted to hydrate as seen in Figure 1(b).

Bulk CPMG $\mathrm{T}_{2}$ distribution measurements readily identified bulk methane gas in the reservoir, as well as water in the pore space, during hydrate formation. As hydrate formation proceeds, the $T_{2}$ lifetime of water in the pore space decreases, and the signal amplitude of this component decreases. This behavior is anticipated for a water wet system where hydrate forms in the pore center 
leaving a residual pore water volume with an increased surface to volume ratio [15-17], and hence enhanced surface relaxation.

(a)
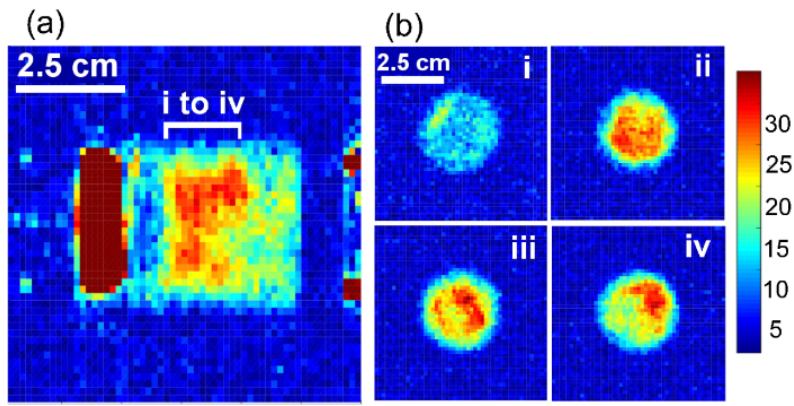

Fig. 2. $2 \mathrm{D}$ slices from $3 \mathrm{D} \pi$-EPI images of $1 \mathrm{H}$ content in the methane hydrate system 26 hours after introducing methane gas. a) The strong methane reservoir signal is off scale at left in the image. No signal is apparent in the interface region of the sand pack. The water distribution is inhomogeneous reflecting a non-uniform distribution of hydrate in the sand pack. b) Four orthogonal slices from the data set of image (a) reveal the transverse inhomogeneity of hydrate formation. High image intensity is high water content and hence low hydrate conversion. The signal from the gas reservoir exceeds the dynamic range of the chosen display scale in Figure 2(a). Signal is simply mapped to the highest intensity of the chosen display range.

The 1D dhk SPRITE [4] profiles reveal that the initial hydrate formation is inhomogeneous longitudinally. Hydrate formation is also inhomogeneous laterally in planes transverse to the direction of gas penetration. 2D Spiral SPRITE [4] measurements and 3D $\pi$-EPI [11] measurements reveal that hydrate formation is greater near the vessel wall. Figure 2 shows $2 \mathrm{D}$ slices from a 3D $\pi$-EPI image of fluid saturation, residual water and pore gas, taken 26 hours after introduction of the gas. The lateral slice shows no signal near the gas head/sand pack interface. This suggests very significant conversion of water to hydrate in this region [20]. SDS is known to promote near complete hydrate formation [19]. Finite signal is observed displaced from the interface. This is due to residual water in the pore space. The lateral slice and the transverse slices in Figure 2(b) show more residual water present in the center of the sand pack and less near the periphery.

Hydrate formation releases heat, increasing the temperature, while the sample vessel wall is in contact with the confining fluid at $4^{\circ} \mathrm{C}$. Hydrate formation may be more favorable near the wall due to heat transfer effects [18]. It has also been reported [19] that adding surfactant to the water phase can create a surface growth pattern during hydrate formation in polymeric vessels. A loose sand pack near the exterior surface may also permit enhanced gas transport and hence enhanced hydrate formation near the sand pack exterior.

One of the main goals of this work was to identify residual fluids present in the pore space as hydrate forms. In addition to residual water in the pore space it is reasonable to posit the presence of unreacted methane gas in the pore space [15]. The methane gas head was maintained at constant pressure throughout the ten days of hydrate formation to ensure an unlimited supply of methane to the system.

Bulk $T_{1}$ measurements undertaken throughout the period of hydrate formation identify the appearance of a long $\mathrm{T}_{1}$ signal component in the sample. The bulk $\mathrm{T}_{1}-\mathrm{T}_{2}$ [12] measurement is ideally suited to distinguishing multiple molecular species present in a sample such as the hydrate system. The long $\mathrm{T}_{1}$ species is not present prior to introduction of methane gas.

Bulk $\mathrm{T}_{1}-\mathrm{T}_{2}$ measurements were undertaken at regular intervals throughout the ten day reaction. Selected results for four different time intervals are shown in Figure 3. Figure 3(a) corresponds to 4 hours after introduction of the gas. The four distinct peaks, or clusters of peaks, may be assigned as follows, peak $\mathrm{P}_{1}$, is bulk methane gas in the gas head. This peak has constant integrated signal and fixed $\mathrm{T}_{1}-\mathrm{T}_{2}$ coordinates throughout the period of hydrate formation. The $T_{1}$ and $T_{2}$ of $P_{1}$ are roughly equivalent suggesting high mobility and a ${ }^{1} \mathrm{H}$ species in the extreme narrowing regime. Peak $\mathrm{P}_{2}$ is bulk-like water found in the apparatus threads, and in the methane gas head at early experimental times. This peak does not change in $\mathrm{T}_{1}-\mathrm{T}_{2}$ coordinates, but it does decrease in amplitude as hydrate formed with residual water present in the gas head.

The cluster of peaks labeled $\mathrm{P}_{3}$ are assigned to water in the pore space. These peaks decrease in amplitude as hydrate formation proceeds but there is no decisive shift observed in the $\mathrm{T}_{1}-\mathrm{T}_{2}$ coordinates. This is due at least in part to the fact there are multiple peaks. Spatially resolved $\mathrm{T}_{2}$ distribution maps determined from 1D SESPI [4] measurement and spatially resolved $\mathrm{T}_{1}-\mathrm{T}_{2}$ measurement unambiguously permit assignment of the $\mathrm{P}_{1}$ and $\mathrm{P}_{2}$ peaks based on their positions in space and their relaxation times. The $T_{1}, T_{2}$ lifetimes of peak $P_{3}$ are consistent with water in the pore space $[21,22]$ and simple control measurements confirm the assignment.

Peak $\mathrm{P}_{4}$ with a very long initial $\mathrm{T}_{1}$, Figure 3 , was not anticipated prior to experiment. The long $\mathrm{T}_{1}$ signal component, on the order of 10 seconds, was confirmed by bulk $\mathrm{T}_{1}$ measurement undertaken as outlined above. $\mathrm{T}_{1}$ lifetimes generally increase with pressure for spin $1 / 2$ gases due to the spin rotation relaxation mechanism [24]. Since it is difficult to unambiguously assign peak $\mathrm{P}_{4}$ to pore gas through a control experiment we must consider alternate hypotheses. We consider first the possibility that $\mathrm{P}_{4}$ is due to (1) water closely associated with the hydrate network or (2) methane gas dissolved in the water phase. The first hypothesis seems unlikely. The integrated signal ratio of peaks $\mathrm{P}_{4} / \mathrm{P}_{3}$ is approximately 
0.30 at four hours of reaction, as observed in Figure 3 (a). Although the residual water saturation was only $27 \%$, there is still significant water present. In a water wet system it is difficult to imagine that a water signal this significant would not appear as part of peak $\mathrm{P}_{3}$.

The second hypothesis must also account for the quantity of material in peak $\mathrm{P}_{4}$. Methane solubility in water is $0.1 \%$ [27-29] dramatically less than the quantity of signal observed in $\mathrm{P}_{4}$. Although SDS is a surfactant and might be considered to promote hydrocarbon solubility, it has been discounted as an aid to methane solubility [28] in hydrate studies. One might imagine supersaturation or Nano bubbles as a means of increasing the quantity of methane gas dissolved in water, but these mechanisms would seem more likely in the case of hydrate dissociation as opposed to hydrate formation. Neither of these hypotheses readily explains the unusual $\mathrm{T}_{1}$ and $\mathrm{T}_{2}$ values of peak $\mathrm{P}_{4}$. A very similar peak appears in analogous hydrate dissociation experiments (data not shown) so a viable hypothesis must work for both formation and dissociation processes.

This leads us to a consideration of unreacted methane gas in the pore space as the third hypothesis. Pore gas (residual gas saturation) denotes the gas saturation in the pore space during hydrate formation [15]. We assume that a small fraction of the pore gas dissolves in the water phase but the remainder remains in the pore space [29]. A 70\% hydrate saturation will significantly occupy the pore space [26] but there is an unlimited supply of methane gas available to the system at 1500 psi.

Important evidence in support of the pore gas hypothesis is the bifurcation in the $T_{1}$ and $T_{2}$ relaxation times of peak $\mathrm{P}_{4}$. Pure methane gas, under the same bulk pressure and temperature conditions, has equivalent $T_{1}$ and $\mathrm{T}_{2}$ as observed in peak $\mathrm{P}_{1}[24,25]$. Methane gas at elevated pressure in microporous solids is known to bifurcate in $T_{1}$ and $T_{2}$ with $T_{2}$ often substantially reduced from $\mathrm{T}_{1}$ [23,24]. Kausik [24] has studied this phenomena in detail in a model porous vycor glass. The reason for the decrease in $T_{2}$ is interaction with the pore surface leading to slower motion [24] and a greater sensitivity to relaxation. The $\mathrm{T}_{1}$ of peak $\mathrm{P}_{4}$ is longer than that of the pure gas species $P_{1}$ in Figure 3 (a). The pore gas hypothesis requires that the pore pressure increases as gas is trapped in the sand pack while expansive hydrate is produced $[16,26]$. Such a pressure increase will increase $\mathrm{T}_{1}$.

As hydrate formation proceeds, peak $\mathrm{P}_{4}$ shifts to shorter $T_{1}$ and is reduced in amplitude as would be expected if the gas pressure in an isolated pore space were decreasing, as further hydrate formed with gas consumed. Figure $3(\mathrm{~d})$ shows that peak $\mathrm{P}_{4}$ has a $\mathrm{T}_{1}$ of 1.4 s after 200 hours. Figure 1(c) shows that water was homogenously distributed in the sand pack at this time. 212 hours after the introduction of the gas, the residual water saturation, Figure 3(d), was $2.8 \%$. This must be close to irreducible water saturation precluding further hydrate formation.

We emphasize that the $T_{1}-T_{2}$ results are semiquantitative since the long $\mathrm{T}_{1}$ value of these species was outside the range anticipated by the bulk $\mathrm{T}_{1}-\mathrm{T}_{2}$ measurement and its timing parameters. The long $\mathrm{T}_{1} \mathrm{~s}$ of peak $\mathrm{P}_{4}$, during the first 26 hours after introduction of the gas, ensures that it will contribute minimally to the signal observed in the dhk SPRITE profiles of Figure 1(b) and the first two profiles in Figure 1(c) [4].

The residual water saturations calculated based on bulk $\mathrm{T}_{1}-\mathrm{T}_{2}$ measurements and 1D dhk SPRITE measurements agree within the first 26 hours of gas introduction. This confirmed that the pore gas magnetization had a minimal contribution to the 1D dhk SPRITE images during early stages of hydrate formation. (a)

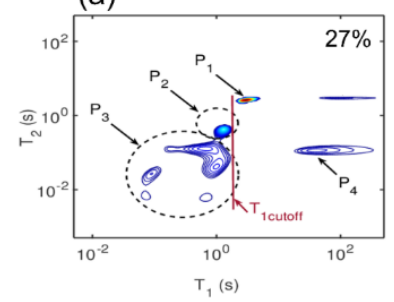

(c)

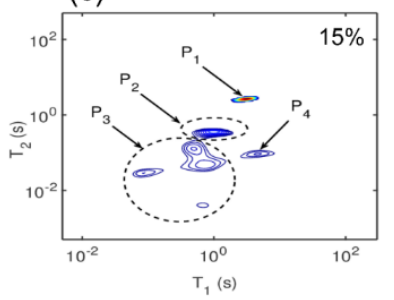

(b)

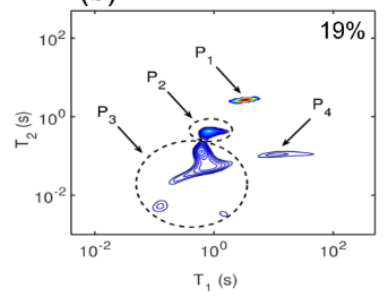

(d)

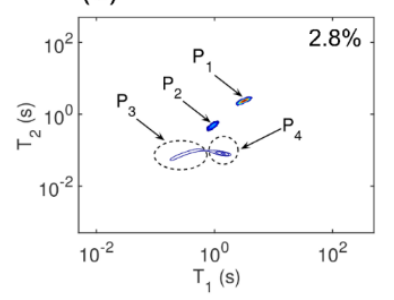

Fig. 3. Bulk T1-T2 measurement of the sand pack as a function of time after methane supply as hydrate forms. In each sub figure the residual water saturation in the pore space is at top right. (a) 4 hours (b) 14 hours, (c) 26 hours and (d) 212 hours. The peak labeled P1 is bulk gas in the gas head, P2 is bulk like water including water in threads of the apparatus, P3 is residual water in the pore space. P4 is assigned to residual gas in the pore space. T1cutoff separates the gas phase peaks from those of water in the system. The P4 peak shifts from long T1 to shorter T1, as hydrate forms. Bulk T1-T2 measurements, represented in Figure 3(a)-(d), have signal to noise ratios of $224,154,150$ and 80 . Variation in the regularization parameter did not change the major characteristics of the peaks [30].

\section{Conclusion}

In this study methane hydrate was formed in a water saturated sand pack, within a MRI compatible metallic core holder, at $1500 \mathrm{psi}$ and $4{ }^{\circ} \mathrm{C}$. 1D dhk SPRITE MRI measurements spatially and temporally monitored 
hydrate formation. 1D dhk SPRITE measurements produced ${ }^{1} \mathrm{H}$ density weighted images for bulk gas, bulk water and water in the pore space during hydrate formation. A 2 hour induction period was observed with methane hydrate formation commencing at the interface of the sand pack and gas head. The highest rate of hydrate formation occurred between 2 hours and 4 hours after introduction of the gas. CPMG $\mathrm{T}_{2}$ measurements suggested that in this water wet system, hydrate occupies the pore center. Intense, rapidly switched magnetic field gradients may initiate hydrate formation, through vibration, as often occurs with conventional phase transitions.

1D dhk SPRITE profiles showed that the initial hydrate formation was inhomogeneous in the longitudinal direction. 3D $\pi$-EPI measurements revealed that hydrate formation was more significant close to the sand pack walls at early experimental times. 3D $\pi$-EPI images suggested a complete, or near complete, conversion of water to hydrate at the sand pack/gas interface. Bulk $\mathrm{T}_{1}-\mathrm{T}_{2}$ measurements quantified the residual water saturation during hydrate formation. Bulk $\mathrm{T}_{1}-\mathrm{T}_{2}$ measurements discriminated the residual water from the pore gas during hydrate formation. The water saturation was $2.8 \%$ after ten days of hydrate formation.

\section{References}

1. E.D. Sloan, C. A. Koh, Clathrate Hydrates of Natural Gases Third edition, CRC Press, Taylor \& Francis Group, New York, 2008, pp. 16-193.

2. G. Ersland, J. Huseb $\varnothing$, A. Graue, B. A. Baldwin, J. Howard, J. Stevens, Measuring Gas Hydrate Formation and Exchange with $\mathrm{CO}_{2}$ in Bentheim Sandstone Using MRI Tomography, Chem. Eng. J., 158 (2010) 25-31.

3. J. C. Stevens, B. A. Baldwin, A. Graue, G. Ersland, J. Husebø, J. J. Howard, Measurements of Hydrate Formation in Sandstone, SCA 2007-36, International Symposium of the Society of Core Analysts, Calgary, Canada, Sept. 10-12, 2007.

4. C. E. Muir, B. J. Balcom, in: G.A. Webb (Ed.), Pure Phase Encode Magnetic Resonance Imaging of Fluids in Porous Media, Annual Reports on NMR Spectroscopy, Academic Press, Burlington, Vol. 77 (2012) 81-113.

5. A. Afrough, M. Shakerian, M. S. Zamiri, B. MacMillan, F. Marcia, B. Newling, L. RomeroZeron, B. J. Balcom, Magnetic Resonance Imaging of High Pressure Carbon Dioxide Displacement: Fluid/Surface Interaction and Fluid Behavior, SPE Journal, SPE-189458-PA,
February 2018, https://doi.org/10.2118/189458PA.

6. P. Wang, S. Wang, Y. Song, M. Yang, Dynamic Measurements of Methane Hydrate Formation/ Dissociation in Different Gas Flow Direction, Appl. Energy (in press), https://doi.org/10.1016/ j.apenergy.2017.08.056.

7. P. Wang, M. Yang, B. Chen, Y. Zhao, J. Zhao, Y. Song, Methane Hydrate Reformation in Porous Media with Methane Migration, Chem. Eng. Sci., 168 (2017) 344-351.

8. S. A. Bagherzadeh, I. L. Moudrakovski, J. A. Ripmeester, P. Englezos, Magnetic Resonance Imaging of Gas Hydrate Formation in a Bed of Silica Sand Particles, Energy Fuels, 25 (7) (2011) 3083-3092.

9. M. Shakerian, B. J. Balcom, An MR/MRI Compatible Core Holder with the RF Probe Immersed in the Confining Fluid, J. Magn. Reson., 286 (2018) 36-41.

10. M. Shakerian, F. Marica, A. Afrough, F. G. Goora, M. Li, S. Vashaee, B. J. Balcom, A High-Pressure Metallic Core Holder for Magnetic Resonance Based on Hastelloy-C, Rev. Sci. Instrum., 88 (2017) 123703.

11. D. Xiao, B. J. Balcom. $\pi$ Echo-Planar Imaging with Concomitant Field Compensation for Porous Media MRI, J. Magn. Reson., 260 (2015) 38-45.

12. S. Vashaee, M. Li, B. Newling, B. MacMillan, F. Marica, H.T. Kwak, J. Gao, A. M. Al-harbi, B. J. Balcom, Local $\mathrm{T}_{1}-\mathrm{T}_{2}$ Distribution Measurements in Porous Media, J. Magn. Reson., 287 (2018) 113-122.

13. H. Ganji, M. Manteghian, K. Sadaghiani zadeh, M. R. Omidkhah, H. Rahimi Mofrad, Effect of Different Surfactants on Methane Hydrate Formation Rate, Stability and Storage Capacity, Fuel, 86 (2007) 434-441.

14. P. Linga, N. Daraboina, J. A. Ripmeester, P. Englezos, Enhanced Rate of Gas Hydrate Formation in a Fixed Bed Column Filled with Sand Compared to a Stirred Vessel, Chem. Eng. Sci., 68 (2012) 617-623.

15. J. Zhao, L. Yang, Y. Liu, Y. Song, Microstructural Characteristics of Natural Gas Hydrates Hosted in Various Sand Sediments, Phys. Chem. Chem. Phys., 17 (2015) 22632.

16. M. B. Clennell, M. Hovland, J. S. Booth, P. Henry, W. J. Winters, Formation of Natural Gas Hydrates in Marine Sediments 1. Conceptual Model of Gas Hydrate Growth Conditioned by Host Sediment Properties, J. Geophys. Res. B, 104. No. B10 (1999) 22985-23003. 
17. S. Gao, W. House, W. G. Chapman, NMR/MRI Study of Clathrate Hydrate Mechanisms, J. Phys. Chem. B, 109 (2005) 19090-19093.

18. R. T. Meindinyo, T. M. Svartaas, T. N. Nordbø, R. Bøe, Gas Hydrate Growth Estimation Based on Heat Transfer, Energy Fuels, 29 (2) (2015) 587-594.

19. J. Yoslim, P. Linga, P. Englezos, Enhanced Growth of Methane-Propane Clathrate Hydrate Crystals with Sodium Dodecyl Sulfate, Sodium Tetradecyl Sulfate, and Sodium Hexadecyl Sulfate Surfactants, J. Cryst. Growth, 313 (2010) 68-80.

20. D. D. David, F. Guerton, C. Dicharry, J. P. Torré, D. Broseta, Hydrate Growth at the Interface between Water and Pure or Mixed $\mathrm{CO}_{2} / \mathrm{CH}_{4}$ Gases: Influence of Pressure, Temperature, Gas Composition and WaterSoluble Surfactants, Chem. Eng. Sci., 132 (2015) 118-127.

21. R. L. Kleinberg, W. E. Kenyon, P. P. Mitra, Mechanism of NMR Relaxation of Fluids in Rock, J. Magn. Reson. SerA., 108 (1994) 206-214.

22. G. R. Coates, L. Xiao, M. G. Prammer, NMR Logging Principles and Applications, Gulf Publishing Company, Houston, 1999, pp. 3389.

23. C. Straley, An Experimental Investigation of Methane in Rock Materials, SPWLA 38th Annual Logging Symposium, 15-18 June, Houston, Texas, 1997.

24. A. Papaioannou, R. Kausik, Methane Storage in Nanoporous Media as Observed via High field NMR Relaxometry, Phys. Rev. Applied, 4 (2015) 024018.

25. C. S. Johnson, J. S. Waugh, Nuclear Relaxation in Gases: Mixtures of Methane and Oxygen, J. Chem. Phys., 35 (6) (1961) 2020-2024.

26. J. Y. Lee, J. C. Santamarina, C. Ruppel, Volume Change Associated with Formation and Dissociation of Hydrate in Sediment, Geochem. Geophys. Geosysm., 11 (3) (2010) 1-13.

27. P. Servio, P. Englezos, Measurement of Dissolved Methane in Water in Equilibrium with its Hydrate, J. Chem. Eng. Data, 47 (2002) 87-90.

28. J. Verrett, D. Posteraro, P. Servio, Surfactant Effects on Methane Solubility and Mole Fraction during Hydrate Growth, Chem. Eng. Sci., 84 (2012) 80-84.

29. I. N. Tsimpanogiannis, P. C. Lichtner, Methane Solubility in Water under Hydrate Equilibrium Conditions: Single Pore and Pore Network
Studies, Proceedings of the 7th International Conference on Gas Hydrates (ICGH 2011), Edinburgh, Scotland, United Kingdom, July 17-21, 2011.

30. Y. Q. Song, L. Venkataramanan, M. D. Hurlimann, M. Flaum, P. Frulla, C. Straley, T ${ }^{-}$ $\mathrm{T}_{2}$ Correlation Spectra Obtained Using a Fast Two-dimensional Laplace Inversion, J. Magn. Reson., 154 (2) (2002) 261-268. 